\title{
Teaching NeuroImages: A rare case of metachromatic leukodystrophy with multiple bilateral cranial nerve enhancement
}

Purabi Sonowal, MD, Dinesh Lulla, MD, Amanda Weber, DO, and Deniz Altinok, MD

Neurology ${ }^{\circledR}$ 2019;93:e1742-e1743. doi:10.1212/WNL.0000000000008400

Figure Imaging

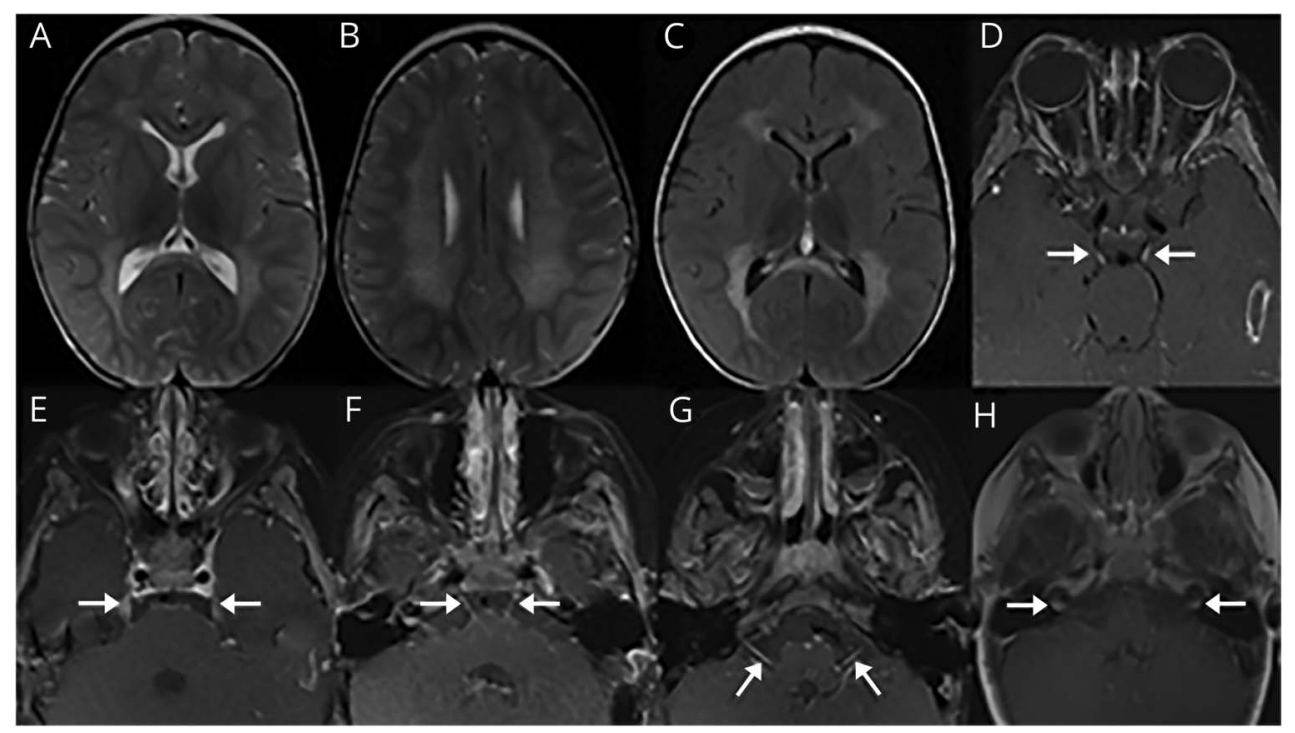

Axial T2 (A, B) and fluid-attenuated inversion recovery (C) images show symmetric confluent hyperintensities in a leopard-like appearance throughout the periventricular white matter, centrum semiovale (sparing subcortical $U$ fibers), genu, and splenium of the corpus callosum. Axial contrast-enhanced T1 images show symmetric enhancement of the bilateral third cranial nerve ( $D$, arrows), bilateral fifth cranial nerve ( $E$, arrows), bilateral sixth cranial nerve $(F$, arrows), bilateral seventh cranial nerve (G, arrows), and bilateral eighth cranial nerve (H, arrows).

A 32-month-old boy, born at term, presented with progressive developmental regression from 14 months of age. On examination, he had horizontal nystagmus, diminished gag reflex, hypertonicity, and depressed reflexes. Brain MRI revealed symmetric T2/fluid-attenuated inversion recovery confluent hyperintensities in the periventricular white matter, corpus callosum, and centrum semiovale (sparing subcortical U fibers) with enhancement of multiple cranial nerves (figure). The characteristic leopard-like appearance of the hyperintensities prompted further evaluation for metachromatic leukodystrophy (MLD) ${ }^{1,2}$ Arylsulfatase A enzyme activity was found to be low, and the patient had a homozygous pathogenic variant in the ARSA gene (c.465+1G), confirming MLD.

\section{Study funding}

No targeted funding reported.

\author{
Correspondence \\ Dr. Sonowal \\ psonowal@dmc.org
}

\section{MORE ONLINE}

$\rightarrow$ Teaching slides

links.lww.com/WNL/

A985 


\section{Disclosure}

The authors report no disclosures relevant to the manuscript. Go to Neurology.org/N for full disclosures.

Appendix Authors

\begin{tabular}{llll}
\hline Name & Location & Role & Contribution \\
\hline $\begin{array}{l}\text { Purabi } \\
\text { Sonowal, } \\
\text { MD }\end{array}$ & $\begin{array}{l}\text { Children's } \\
\text { Hospital of } \\
\text { Michigan, } \\
\text { Detroit }\end{array}$ & Author & $\begin{array}{l}\text { Study concept and design; } \\
\text { acquisition, analysis, drafted } \\
\text { the manuscript for intellectual } \\
\text { content }\end{array}$ \\
\hline $\begin{array}{l}\text { Dinesh } \\
\text { Lulla, MD }\end{array}$ & $\begin{array}{l}\text { Children's } \\
\text { Hospital of } \\
\text { Michigan, } \\
\text { Detroit }\end{array}$ & Author & $\begin{array}{l}\text { Interpreted the data, revised } \\
\text { the manuscript for intellectual } \\
\text { content }\end{array}$ \\
& &
\end{tabular}

Appendix (continued)

\begin{tabular}{llll}
\hline Name & Location & Role & Contribution \\
\hline $\begin{array}{l}\text { Amanda } \\
\text { Weber, } \\
\text { Do }\end{array}$ & $\begin{array}{l}\text { Children's } \\
\text { Hospital of } \\
\text { Michigan, } \\
\text { Detroit }\end{array}$ & Author & $\begin{array}{l}\text { Interpreted the data, revised } \\
\text { the manuscript for intellectual } \\
\text { content }\end{array}$ \\
\hline $\begin{array}{l}\text { Deniz } \\
\begin{array}{l}\text { Altinok, } \\
\text { MD }\end{array}\end{array}$ & $\begin{array}{l}\text { Children's } \\
\text { Hospital of }\end{array}$ & Author & Acquisition of data \\
\hline
\end{tabular}

\section{References}

1. Singh RK, Leshner RT, Kadom N, Vanderver AL. Isolated cranial nerve enhancement in metachromatic leukodystrophy. Pediatr Neurol 2009;40:380-382.

2. Maia AC Jr, da Rocha AJ, da Silva CJ, Rosemberg S. Multiple cranial nerve enhancement: a new MR imaging finding in metachromatic leukodystrophy AJNR Am J Neuroradiol 2007;28:999. 


\section{Neurology}

\section{Teaching NeuroImages: A rare case of metachromatic leukodystrophy with multiple bilateral cranial nerve enhancement}

Purabi Sonowal, Dinesh Lulla, Amanda Weber, et al.

Neurology 2019;93;e1742-e1743

DOI 10.1212/WNL.0000000000008400

This information is current as of October 28, 2019

\section{Updated Information \&} Services

\section{References}

Subspecialty Collections

Permissions \& Licensing

Reprints including high resolution figures, can be found at: http://n.neurology.org/content/93/18/e1742.full

This article cites 2 articles, 0 of which you can access for free at: http://n.neurology.org/content/93/18/e1742.full\#ref-list-1

This article, along with others on similar topics, appears in the following collection(s):

All Genetics

http://n.neurology.org/cgi/collection/all_genetics

Clinical neurology history

http://n.neurology.org/cgi/collection/clinical_neurology_history

Developmental disorders

http://n.neurology.org/cgi/collection/developmental_disorders

Metabolic disease (inherited)

http://n.neurology.org/cgi/collection/metabolic_disease_inherited MRI

http://n.neurology.org/cgi/collection/mri

Information about reproducing this article in parts (figures,tables) or in its entirety can be found online at:

http://www.neurology.org/about/about_the_journal\#permissions

Information about ordering reprints can be found online:

http://n.neurology.org/subscribers/advertise

Neurology ${ }^{\circledR}$ is the official journal of the American Academy of Neurology. Published continuously since 1951, it is now a weekly with 48 issues per year. Copyright (O 2019 American Academy of Neurology. All rights reserved. Print ISSN: 0028-3878. Online ISSN: 1526-632X.

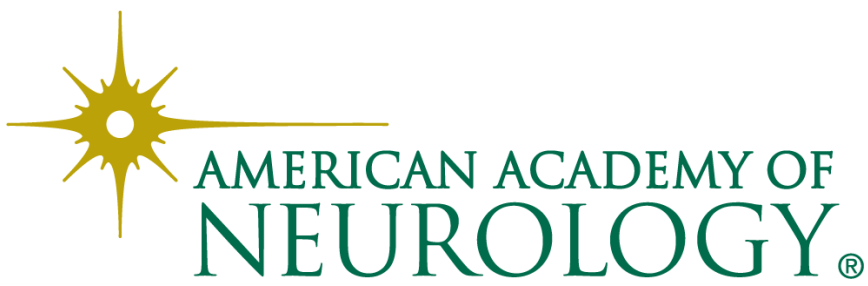

